\title{
Der Better-Life-Index Jugend: Was kann er für eine evidenzbasierte Jugendpolitik leisten?
}

\begin{abstract}
Kathrin Gärtner ${ }^{\star}$ und Ivo Ponocny ${ }^{\star *}$
Zusammenfassung

Im Rahmen des 7 . Berichts zur Lage der Jugend in Österreich wurde unter Einbeziehung der Zielgruppe und von Expert/innen ein Index zur Lebensqualität von jungen Menschen entwickelt, berechnet und vorgestellt, der Better-Life-Index Jugend. Dabei handelt es sich um einen Summenindex basierend auf der Erhebung EUSILC 2013, der aus zehn Subindizes zu den Lebensbereichen materielle Lebensbedingungen, Arbeit, Gesundheit, Bildung, soziale Beziehungen, Freizeit, Sicherheit, Qualität der gesellschaftlichen Organisation, Wohnen und subjektives Wohlbefinden besteht und sich vor allem dazu eignet, Gruppenunterschiede bezüglich verschiedener Lebensbereiche zu analysieren und Risikogruppen zu identifizieren. So zeigt sich beispielsweise, dass Jugendliche auf dem Land in fast allen Lebensbereichen höhere Werte aufweisen als Jugendliche in der Stadt. Was der Index aufgrund seiner Konstruktionsweise jedoch nicht leisten kann, ist ein Vergleich verschiedener Lebensbereiche, das Aufzeigen einer Entwicklung über die Zeit oder ein Vergleich mit anderen Ländern. Für solche Zwecke müsste ein entsprechender Index ganz oder zumindest teilweise auf Grundlage anderer Datenquellen aufgebaut werden.
\end{abstract}

Schlagwörter: Jugend, Lebensqualität, zusammengesetzte Indikatoren, Politik

The Better Life Index Youth: What can it contribute to an evidence-based youth policy? Abstract

For the $7^{\text {th }}$ report on the situation of youth in Austria, a Better Life Index Youth was constructed involving the participation of youth experts and young people themselves with the aim to measure youth well-being in Austria. The Better Life Index Youth is a sum index based on data from EU-SILC 2013 and consisting of ten sub-indices: material living conditions, work, health, education, social relations, leisure, safety, quality of societal organisation, housing and subjective well-being. The index is especially suitable to analyse group differences in different domains and therefore to identify risk populations. It could be shown, for example, that young people living in rural areas generally reach higher values than young people living in the cities. However, the BLI Youth is currently no suitable measure to compare different life domains, to show developments over time or to enable international comparisons. An index which can serve these needs would at least partly have to be based on other data.

Keywords: Youth, Well-being, Composite Indicators, Policy

\footnotetext{
${ }^{\star}$ Kathrin Gärtner, Fachhochschule Wiener Neustadt. E-Mail: kathrin.gaertner@fhwn.ac.at

**Ivo Ponocny, MODUL University Vienna. E-Mail: ivo.ponocny@modul.ac.at

Dieser Artikel entstand im Zusammenhang mit der Arbeit der Autor/inn/en am 7. Bericht zur Lage der Jugend in Österreich, welcher vom Bundesministerium für Familie und Jugend beauftragt wurde. Die in diesem Artikel geäußerten Ansichten reflektieren jedoch nicht notwendigerweise die Überzeugungen der beteiligten Institutionen MODUL University Vienna, Statistik Austria, Donau-Universität Krems oder BMFJ bzw. der aktuellen Affiliation der Erstautorin Fachhochschule Wiener Neustadt. Die Autorin und der Autor danken Manfred Zentner für zentrale Beiträge und Hinweise zum Text, Vivien Unger und Bernhard Saupe für die kritische Durchsicht sowie den beiden anonymen Gutachter/innen für ihre wertvollen Anmerkungen
} 


\section{Einleitung}

Gemäß Nationalratsentschluss vom 28.9.1988 hat das jeweils zuständige Ministerium in jeder Legislaturperiode dem Nationalrat einen Bericht zur Lage der Jugend in Österreich vorzulegen. In der XXV. Gesetzgebungsperiode sollte im Rahmen des 7. Jugendberichts auch die Konzeption, Berechnung und Analyse zu einem BetterLife-Index Jugend (im Folgenden BLI Jugend) nach Vorbild des Better-Life-Index der OECD (OECD 2011, 2015) erfolgen. Bereits in der Ausschreibung war sowohl die Einbindung einschlägiger Expert/innen (Vertreter/ innen verschiedener Jugendorganisationen und -vertretungen sowie der Jugendhilfe) sowie von Jugendlichen selbst festgelegt. Ursprünglich sah die Ausschreibung auch die Durchführung einer eigenen Befragung zur Füllung von Datenlücken von mindestens 800 Jugendlichen vor, das letztendlich beauftragte Konsortium aus Donau-Universität Krems/Department für Migration und Globalisierung, Statistik Austria und der MODUL University Vienna entschied sich aber dafür - nicht zuletzt aus Kostengründen - keine eigene Erhebung durchzuführen, sondern eine Index-Berechnung auf Basis der österreichischen Daten von EU-SILC aus dem Jahr 2013 anzubieten, da hier im Rahmen eines Lebensqualitäts-Sondermoduls bereits ein umfangreiches Set an Indikatoren zu Lebensqualität und Lebensbedingungen vorlag.

Die konkreten Zielsetzungen des Ministeriums für Familie und Jugend hinter der Beauftragung der Konstruktion eines Better-Life-Index Jugend sind nicht dokumentiert (etwas, was beispielsweise von Babic (2017) kritisiert wurde). Die Tatsache, dass der Jugendbericht bereits in der Mitte der Legislaturperiode beauftragt wurde, lässt aber darauf schließen, dass man sich hiervon relevante Informationen erhoffte, die als Leitfaden der weiteren Politik dienen können.

Evidenzbasierte Politikgestaltung gilt in Österreichs und Europas Jugendpolitik nicht nur als Notwendigkeit, sondern - auch in Österreich spätestens mit der Einführung der Österreichischen Jugendstrategie - als Standard. In der aktuellen EU-Jugendstrategie „Investitionen und Empowerment", die von 2010 bis 2018 gültig ist, wird klargestellt, dass „fundierte Sachkenntnis [...] eine Voraussetzung für eine schlüssige Politik“ ist (European Commission 2009). Auch in der Agenda 2020 des Europarats, in der dessen Jugendpolitik definiert wird und die eine Aufforderung an die Mitgliedsstaaten darstellt, sich gezielt der Rechte, der Inklusion und der Zukunftsmöglichkeiten der Jugendlichen anzunehmen, wird festgehalten, dass Jugendforschung eine Priorität für die Entwicklung der Jugendpolitik darstellt. Dazu wird vorgeschlagen, laufend Studien durchzuführen und die Kooperation zwischen Jugendforschung und Politikgestaltung zu intensivieren (Council of Europe 2008). Auch im Youth Policy Manual (Denstat 2009) wird speziell auf die Bedeutung von Jugendforschung für evidenzbasierte Jugendpolitikgestaltung hingewiesen, aber auch auf die Bedeutung der Erfahrungen von Praktiker/ inne/n. Datenbanken zu Jugendpolitik, Indikatoren bei Eurostat oder der alle drei Jahre erscheinende Europäische Jugendbericht sind Beispiele für Faktensammlungen als Basis für Politikentwicklung im Jugendbereich.

Was die konkrete Berücksichtigung von Lebenszufriedenheit in der internationalen evidenzbasierten Politikgestaltung angeht, ist eine umfassende Ausrichtung der politischen Planung an den Ergebnissen relevanten Social Reportings zwar nach wie vor nicht gegeben (siehe Bache/Reardon 2016), allerdings gibt es dennoch eine Vielzahl von Beispielen für die Verwendung von lebensqualitätsbezogenen Indikatoren als Entscheidungsgrundlage bzw. als Zielkriterium. Noll (2004) erwähnt dabei insbesondere Entwicklungen hin zu regionalen Lebensqualitätsinitiativen auf Basis von Community Indicators sowie die Konstruktion von populationsspezifischen Indikatoren wie eben Indizes für bestimmte Alters- oder auch Bedarfsgruppen. Viele der von Noll erwähnten Indikatorprojekten, wie zum Beispiel das Projekt „Redefining progress“, in dem für 200 Städte und Gemeinden in den USA Lebensqualitätsindikatoren entwickelt wurden, oder ein Projekt zur Lebensqualität in „Big Citys“ in Neuseeland werden inzwischen nicht mehr oder nur in abgewandelter Form weitergeführt, dafür gibt es inzwischen auch in Österreich regionale oder zielgruppenspezifische Projekte, zum Beispiel zur Lebensqualität in der Stadt Salzburg (siehe Keul et al. 2017) oder von Heimbewohner/innen (siehe Schneider/Kleindienst 2016). Besonders gezielte Maßnahmen sind in Großbritannien zu verzeichnen. Hier hat eine umfassende Orientierung der Politik an Indikatoren der subjektiven Lebensqualität etwa zur Ausweitung des Zugangs zu psychotherapeutischer Behandlung oder zur Etablierung des Care Act beigetragen (siehe wieder Bache/Reardon 2016).

Indikatorensets und Indizes, die sich speziell auf das Wohlbefinden von Jugendlichen und/oder Kindern beziehen, existieren sowohl auf internationaler Ebene als auch für einzelne Länder. Beispiele für nationale Initiativen sind der brasilianische Youth Well-being Index (dell'Aglio et al. 2007) sowie ein von Land, Lamb 
und Mostillo (2001) für die USA entwickelter Index für Kinder- und Jugendlichen-Well-being. Auf globaler Ebene sind der Global Youth Well-being Index (Golding et al. 2014: 9), der Child Well-being Index (CWI) (siehe Foundation for Child Development 2013) sowie die UNICEF-Studie „Child well-being in rich countries“ (UNICEF Office of Research 2013) zu nennen. Was die Einbindung der Zielgruppe betrifft, finden sich nur in der UNICEF-Studie Ansätze expliziter Befragung von Jugendlichen darüber, was ihnen im Leben wichtig ist (Bradshaw et al. 2007). Alle anderen genannten Arbeiten berufen sich in der Konstruktion der Indizes auf theoretische Überlegungen, auf die jeweils verfügbaren Daten oder auf Expertisen von Erwachsenen.

Der Better-Life-Index Jugend wurde wie erwähnt explizit mit dem Auftrag entwickelt, die Zielgruppe einzubinden und ihre Vorstellungen von einem „guten Leben“" zu berücksichtigen. Der folgende Artikel berichtet über das Konzept sowie über die Konstruktionsphasen des BLI Jugend, die damit verbundenen grundlegenden Fragen bzw. die entsprechenden Entscheidungsschritte und stellt ausgewählte Ergebnisse dar, bevor auf mögliche Einsatzfelder und Beschränkungen des BLI Jugend eingegangen und analysiert wird, inwiefern der BetterLife-Index Jugend als geeignetes Instrument für eine evidenzbasierte Jugendpolitik angesehen werden kann.

\section{Ablauf des Projekts}

Der Prozess der Arbeiten am BLI Jugend gestaltete sich wie folgt: Zunächst wurden zentrale Grundsatzentscheidungen zum Index getroffen, dann wurde in einer Vorstudie, die vom Bundesnetzwerk österreichischer Jugendinfos (BÖJI) durchgeführt wurde, in Selbsteinschätzung erhoben, welche Lebensbereiche jungen Menschen bezüglich ihrer persönlichen Lebensqualität wie wichtig sind. Die Ergebnisse dieser Vorstudie wurden dann Gruppen von Jugendlichen und Expert/inn/en bzw. Praktiker/inne/n aus dem Jugendbereich vorgelegt. Aus ihren Einschätzungen wurden in einem iterativen Verfahren die Rechenregeln zur Konstruktion des Better-Life-Index Jugend entwickelt. Nach Berechnung des BLI Jugend wurden schließlich Analysen zur Sensitivität des Instruments durchgeführt.

\subsection{Methodische Festsetzungen}

Zwei methodische Entscheidungen wurden bereits bei der Konzepterstellung getroffen: dass das Lebensqualitätskonzept des Indikatoren-Projekts „Wie geht's
Österreich?" (siehe z.B. Statistik Austria 2016) von Statistik Austria verwendet werden soll (zumindest als Ausgangspunkt für die Diskussion) und dass EU-SILC 2013 als Datenbasis herangezogen wird.

Bei „Wie geht's Österreich?“ handelt es sich um ein Indikatorenprojekt zur Messung von Wohlstand und Fortschritt in Österreich in der Tradition der Initiative „Beyond GDP“ der Europäischen Kommission ${ }^{1}$ und des Stiglitz-Berichts (Stiglitz et al. 2010), das dem Bruttoinlandsprodukt dreißig Indikatoren aus den Bereichen materielle Lebensbedingungen, Lebensqualität und Umwelt zur Seite stellt. Die Lebensqualität wurde dabei bis 2016 durch Indikatoren aus den Lebensbereichen materielle Lebensbedingungen, Arbeit, Gesundheit, Bildung, soziale Teilhabe, Freizeit, Sicherheit, Qualität der gesellschaftlichen Organisation, natürliche Wohnumgebung sowie subjektives Wohlbefinden abgedeckt. Bei der Auswahl dieser zehn Dimensionen (eigentlich 9+1, die subjektive Lebensqualität wurde als konzeptionelle Klammer um alle anderen Lebensbereiche verstanden) orientierte man sich an den 8+1 Dimensionen der Lebensqualität bei Eurostat (Eurostat 2011).

Ein solches System als Ausgangspunkt für die Indexkonstruktion zu verwenden, hat den Vorteil, dass an etablierte Indikatorensysteme und Konzepte angeknüpft werden kann - auch wenn hier kein theoretisch etabliertes System vorliegt -, aber den Nachteil, dass die an der Indexbildung beteiligten Personen (hier Jugendliche und Praktiker/innen aus dem Jugendbereich) in ihren Entscheidungen und dem Ausdruck ihrer Präferenzen vermutlich gelenkt und beeinflusst werden. Tatsächlich wurde dann letztendlich in diesen Gruppen die Entscheidung getroffen, im Sinne der Vergleichbarkeit mit bereits vorhandenen Daten und Konzepten den Index aus zehn Subindizes zu bilden, angelehnt an die oben genannten Bereiche.

Die zweite grundsätzliche Entscheidung betraf die Datengrundlage. Wie bereits erwähnt, wurde bei der Konzeption die Entscheidung getroffen, keine eigene Erhebung als Basis für die Indexberechnung durchzuführen, sondern die Erhebung EU-SILC 2013 (Community Statistics of Income and Living Conditions, siehe z.B. Statistik Austria 2014) zu verwenden, in deren Rahmen in diesem Jahr international ein Sondermodul zum Thema Lebensqualität durchgeführt wurde. Darüber hinaus wurden 2013 auf nationaler

1 http://ec.europa.eu/environment/beyond_gdp/ index_en.html, [12.11.2018] 
Ebene einige zusätzliche Items zu besonderen Feldern der Lebensqualität abgefragt, was diesen Datensatz insgesamt als geeignete Datenbasis erscheinen ließ.

Bei EU-SILC handelt es sich um eine europaweit durchgeführte Studie, die vor allem auf die Bereitstellung von Indikatoren zu Armut und materiellen Lebensbedingungen ausgerichtet ist. Sie enthält darüber hinaus aber auch Items zu Gesundheit, Bildung und subjektivem Wohlbefinden. In Österreich werden dafür jährlich etwa 6000 Haushalte befragt.

Eine Studie in diesem Umfang und dieser Qualität für die Indexbildung heranzuziehen, hat mehrere Vorteile. Zum einen enthält die untersuchte Stichprobe mit insgesamt 1748 Personen im Alter zwischen 16 und 29 (die auch die Fragen des Sondermoduls beantwortet haben) deutlich mehr als die in der Ausschreibung geforderten 800 Jugendlichen, was eine tiefer gegliederte Auswertung auch für Subgruppen ermöglicht. Zum anderen erfüllt EU-SILC als internationale Erhebung der amtlichen Statistik hohe Qualitätskriterien, sowohl was den Stichprobenrahmen (Stichprobe direkt aus dem Zentralen Melderegister) und die Stichprobenziehung als auch was Gewichtung, Hochrechnung und Imputation betrifft. Diese Qualität hätte in einer eigenen Erhebung nicht (was die Stichprobengrundlage anbelangt) oder nur mit unverhältnismäßig hohem Aufwand erreicht werden können. Im Gegensatz zu einem Vorgehen, bei dem existierende Daten mit Ergebnissen einer neuen Erhebung zusammengeführt werden müssen, kann hier für jede Person in der Stichprobe ein eigener Indexwert berechnet werden, was den Vergleich zwischen verschiedenen Gruppen ermöglicht. Dies ist beim Better-Life-Index der OECD zum Beispiel nicht möglich; hier werden globale Länderwerte verwendet.

Als nachteilig angesehen werden muss, dass EUSILC zwar für die Erfassung von Einkommen und Lebensbedingungen im Allgemeinen, nicht aber zum speziellen Zweck der Erstellung eines Jugend-Indexes konstruiert wurde, und dass die Gestalt der Fragen auch in weiten Teilen gesetzlichen bzw. internationalen Vorgaben folgt, die wenig nationalen Spielraum lassen. Daraus ergibt sich, dass die bestehende Erhebung ggf. nicht alle interessierenden Lebensbereiche abdecken kann, keine Änderungen bei Fragebogengestaltung bzw. Itemformulierung möglich sind, dass unterschiedliche Frage- und Antwortformate vorliegen und somit Lebensbereiche nur eingeschränkt vergleichbar erhoben werden können (vgl. dazu Abschnitt 5).
2.2 Befragung der Jugendinfos: Was ist Jugendlichen wichtig?

In einer (nicht repräsentativen) Befragung des Bundesnetzwerks österreichischer Jugendinfos von 1691 Jugendlichen und jungen Erwachsenen zwischen 15 und 30 Jahren, die teils online, teils mit Laptops im öffentlichen Raum durchgeführt wurde, wurde die subjektive Wichtigkeit von Lebensbereichen erfasst. Hätte sich hier herausgestellt, dass einzelne Lebensbereiche bzw. Lebensbedingungen und Gegebenheiten als vergleichsweise unwichtig für das Wohlbefinden angesehen werden, hätte das den Expert/inn/engruppen als Entscheidungsgrundlage dienen können, entsprechende Items aus EU-SILC bei der Index-Konstruktion nicht zu berücksichtigen. Tatsächlich hat sich aber ergeben, dass alle abgefragten Lebensbereiche von der Mehrzahl der teilnehmenden Jugendlichen und jungen Erwachsenen als sehr wichtig oder wichtig angesehen wurden.

Konkret war der Fragebogen so konstruiert, dass zu jedem Item oder jedem (zusammengesetzten) Indikator aus SILC eine entsprechende Frage zur subjektiven Wichtigkeit gestellt wurde. Lautete das SILC-Item etwa „Wie schätzen Sie Ihre Gesundheit ein?", so wurde in der Befragung der Jugendinfos gefragt: „Wie wichtig ist es für dich, in deinem Leben gesund zu sein?" Insgesamt enthielt der Fragebogen, neben einigen soziodemographischen Fragen, 44 Items, für die jeweils die Antwortkategorien ,sehr wichtig,, wichtig, ,weniger wichtig' und, gar nicht wichtig' angeboten wurden. Auch wenn, wie bereits erwähnt, alle Bereiche von der Mehrheit der Teilnehmer/innen entweder als wichtig oder sehr wichtig angesehen wurden, lassen sich doch Unterschiede feststellen: Während Items zu Gesundheit, Glück und Autonomie als besonders bedeutsam eingeschätzt wurden, scheinen eine ruhige Wohnumgebung und ein kurzer Arbeitsweg sowie ein hohes Gehalt jungen Menschen etwas weniger wichtig zu sein. Diese Ergebnisse sind in die Gewichtung der einzelnen Variablen durch die Zielgruppe und durch die Expert/inn/ en eingeflossen.

\subsection{Workshops mit Expert/inn/en zur Indexkon- struktion}

Die genaue Festlegung, welche Items und Variablen überhaupt in den Index aufgenommen werden, welchen Lebensqualitätsdimensionen sie zugeordnet und wie diese gewichtet werden sollten, wurde in iterativen Workshops mit Expert/inn/en aus den Feldern 
Tabelle 1: Variablen der Dimension, Subjektives Wohlbefinden“

\begin{tabular}{ll}
\hline \multicolumn{2}{l}{ Dimension ,Subjektives Wohlbefinden" } \\
\hline $3 / 16$ & glücklich sein \\
$3 / 32$ & "dass das, was ich im Leben mache, wertvoll und nützlich ist" \\
$3 / 32$ & "dass sich das, was ich im Leben mache, lohnt” \\
$3 / 16$ & allgemeine Lebenszufriedenheit \\
$3 / 16$ & optimistisch in die Zukunft blicken können \\
$4 / 16$ & Autonomie \\
\hline
\end{tabular}

Quelle: BMFJ 2016

Jugendarbeit, Gesundheitsförderung, Jugendpolitik und Jugendhilfe getroffen. Zeitlich versetzt fanden Workshops (und zwei Einzelgespräche) mit Gruppen von Jugendlichen und jungen Erwachsenen statt, von denen angenommen wurde, dass sie in der Befragung der Jugendinfos nicht erreicht werden konnten. Dies waren im Einzelnen: junge Menschen mit Migrationshintergrund, sozial benachteiligte Jugendliche, junge Menschen mit körperlicher Behinderung, junge Hochbegabte und Jugendliche am Land (ursprünglich als „regional benachteiligte Jugendliche“ beschrieben, wobei diese Benachteiligungshypothese, wie in 3.3 noch $\mathrm{zu}$ sehen sein wird, so nicht aufrechterhalten werden kann). Auch in diesen Gruppen wurden die Zuordnung von Items zu Dimensionen und ihre Gewichtung diskutiert. Hierbei wurde von einigen der Jugendlichen und jungen Erwachsenen die Auffassung vertreten, dass Gesundheit besonders wichtig sei (was sich ja bereits in der Befragung der Jugendinfos ergeben hatte) und entsprechend höher gewichtet werden müsse. Diese Ergebnisse wurden den Workshops mit erwachsenen Expert/ inn/en regelmäßig zurückgemeldet und flossen so auch direkt in die Indexbildung mit ein. Bezüglich der Frage einer potenziellen Höhergewichtung der Gesundheit waren die erwachsenen Expert/inn/en jedoch eher skeptisch und vertraten die Auffassung, dass die befragten Jugendlichen hier möglicherweise sozial erwünschte Antworteten gegeben hätten, ohne selbst davon wirklich überzeugt zu sein. Da darüber hinaus objektiv nicht klärbar war, um wieviel höher diese Dimension gewichtet werden könnte, wurde die Entscheidung getroffen, alle Dimensionen gleich zu gewichten, dem Subindex Gesundheit jedoch einen besonderen Stellenwert in der Auswertung einzuräumen und so seiner besonderen Hervorhebung durch einige befragte Jugendliche und junge Erwachsene Rechnung zu tragen.

In den Erwachsenen-Workshops wurde jedoch nicht nur darüber diskutiert, wie der Index aufgebaut sein soll, sondern auch wie er konstruiert werden könnte, wäre die Datenverfügbarkeit eine andere.
Bemängelt wurde dabei vor allem das Fehlen von Daten zu den Bereichen Qualität der Ausbildung, Erreichbarkeit und Leistbarkeit von Freizeitangeboten, Sexualität, Umwelt, Nachhaltigkeit und sozialer Gerechtigkeit.

2.4 Ergebnisse der Gruppendiskussionen/Berechnung des Index

Berechnet wurde der Index als Summenindex, wobei für jede Dimension/jeden Subindex maximal zehn Punkte erreicht werden können. Diese werden entsprechend der in den Workshops erarbeiteten Gewichtungsschlüssel auf die einzelnen Items aufgeteilt (zur genauen Berechnung anhand der Gewichte siehe BMFJ 2016, 38ff.).

Den Jugendexpert/inn/en und Praktiker/inne/n wurde in den ersten Workshops eine Startpartition für die Zuordnung von Items zu den Dimensionen vorgeschlagen. Diese wurde weitgehend als schlüssig und nachvollziehbar angesehen. Einzelne Items, wie zum Beispiel das Vertrauen in andere, wanderten im Verlauf der Workshops durch einige Dimensionen (um in diesem Fall schließlich bei der Sicherheit zu landen). Zeitweise wurde auch diskutiert, für einzelne Variablen eine zusätzliche Dimension zu bilden. So wurde beispielsweise in Erwägung gezogen, Autonomie, die ja auch von den Jugendlichen als sehr wichtig erachtet wurde, als eigene Dimension vorzusehen, was jedoch wieder verworfen wurde.

Änderungen bei den Benennungen und damit den Schwerpunktsetzungen der Dimensionen gab es jedoch durchaus. So wurde aus der Dimension ,Natürliche Wohnumgebung',Wohnen und natürliche Wohnumgebung' und aus ,Soziale Teilhabe' ,Soziale Beziehungen. Zur sozialen Teilhabe im engeren Sinne sind in EUSILC gar keine Indikatoren enthalten (eine ebenfalls diskutierte Datenlücke).

Sehr genau diskutiert wurden die Aufteilungen der Gewichte innerhalb der einzelnen Dimensionen. Tabelle 1 zeigt eine solche exemplarisch: das subjektive 
Abbildung 1: Verschieden konstruierte Indizes nach Alter

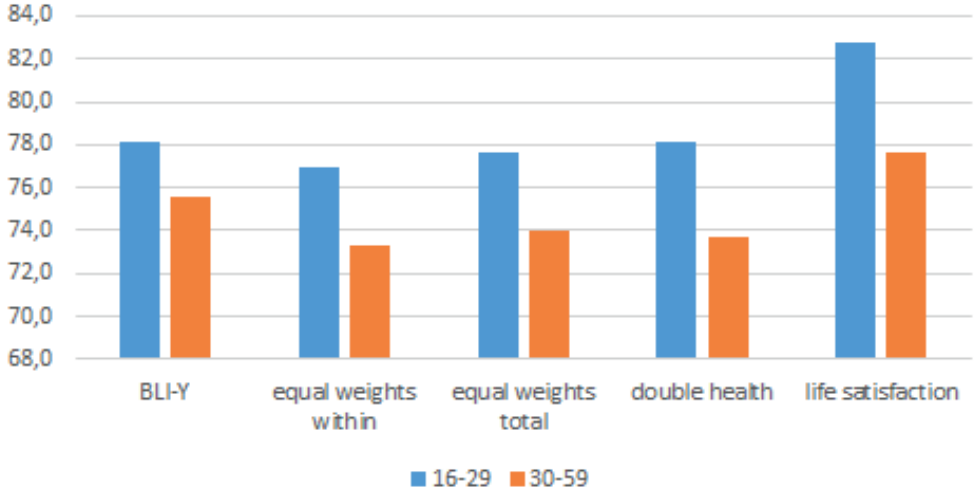

Quelle: Eigene Berechnung und Darstellung auf Basis von EU-SILC 2013, Statistik Austria.

Wohlbefinden, dem letztlich auch die Autonomie zugeordnet wurde.

Für alle weiteren Dimensionen sei auf den Bericht verwiesen (siehe BMFJ 2016, 48ff.), der online frei zur Verfügung steht. Darüber hinaus kann bei Statistik Austria auch eine Berechnungssyntax für den Gesamtindex angefordert werden.

\section{5 Überprüfung der Sensitivität der Ergebnisse in Hinblick auf die Indexgewichte}

Bei diesem Zugang besteht die Gefahr, dass bei unterschiedlichen Zusammensetzungen der jeweiligen Fokusgruppen unterschiedliche Gewichte resultieren, welche ihrerseits $\mathrm{zu}$ unterschiedlichen und damit letztlich willkürlichen Ergebnissen führen könnten. Um dies empirisch zu prüfen, wurde eine Reihe von Simulationen anders erstellter BLI gerechnet: ein Index, in dem alle Variablen innerhalb ihrer jeweiligen Dimensionen gleich gewichtet werden (equal weights within), ein Index, in dem alle Variablen gleich gewichtet werden, unabhängig von der Dimension (equal weights total), ein Index, in dem die Dimension Gesundheit doppelt gewichtet wurde, wie von den jugendlichen Expert/inn/en teilweise vorgeschlagen (double health) und zum Vergleich noch die allgemeine Lebenszufriedenheit, hochskaliert auf 100 Punkte (life satisfaction). Abbildung 1 zeigt allerdings, dass beispielsweise der Vergleich zweier Altersgruppen im Wesentlichen dieselben Resultate liefert, unabhängig von der Gewichtungsvariante, und dasselbe gilt für Geschlecht, Urbanisierungsgrad sowie alle anderen im Jugendbericht vorgenommenen Vergleiche. Die Resultate erscheinen also als recht robust gegenüber methodischen Detailentscheidungen.

\section{Ausgewählte Ergebnisse}

Laut des auf diese Weise konstruierten Indexes weisen junge Menschen in den meisten Lebensbereichen höhere Werte auf als Personen des mittleren Erwachsenenalters. Innerhalb der Gruppe der Jugendlichen und jungen Erwachsenen ergeben sich für Personen mit Migrationshintergrund, für arbeitslose junge Menschen und für junge Erwachsene $\mathrm{ab} 20$ ohne einen über die Pflichtschule hinausgehenden Schulabschluss in fast allen Lebensbereichen niedrigere Werte. Als weitere Risikofaktoren haben sich ein schlechter Gesundheitszustand und ein niedriges äquivalisiertes Haushaltseinkommen erwiesen. Junge Frauen und junge Männer weisen in Bezug auf den Gesamt-BLI keine signifikanten Unterschiede auf.

Im Folgenden sollen die Unterschiede zwischen jungen Menschen und Personen des mittleren Erwachsenenalters, zwischen jungen Männern und jungen Frauen sowie zwischen jungen Menschen aus Stadt und Land näher beleuchtet werden, um exemplarisch $\mathrm{zu}$ zeigen, welche Erkenntnisse durch einen solchen Index ermöglicht werden. Dafür wurden teilweise zusätzliche statistische Analysen durchgeführt, die sich in dieser Form nicht im Jugendbericht finden.

\subsection{Risikophase Jugend?}

Jugendliche werden einerseits als besonders gefährdete und problembehaftete Gruppe angesehen, andererseits als jene Gruppe, die tatsächlich die Zukunft gestalten wird und daher eine besonders politikrelevante ist. In Tabelle 2 findet sich nun ein Vergleich zwischen den Werten der jungen Erwachsenen und den Werten, die sich ergeben, wenn man für Menschen des mittleren 
Tabelle 2: BLI Jugend und Subindizes nach Altersgruppen

\begin{tabular}{|c|c|c|c|c|}
\hline & $\begin{array}{c}16-29 \\
(n=1876)\end{array}$ & $\begin{array}{c}30-59 \\
(n=5729)\end{array}$ & $\begin{array}{l}\text { Effect size } \\
\text { (Cohen's d) }\end{array}$ & $\begin{array}{c}\text { p-Wert (t-Test für un- } \\
\text { abh. Stichproben) }\end{array}$ \\
\hline BLI & 77,0 & 74,6 & 0,22 & $<0,001$ \\
\hline Materielle Lebensbedingungen & 6,8 & 7,1 & $-0,15$ & $<0,001$ \\
\hline Produktive Aktivitäten und Arbeit & 8,4 & 8,4 & $<0,01$ & 0,952 \\
\hline Gesundheit & 8,4 & 7,4 & 0,49 & $<0,001$ \\
\hline Soziale Beziehungen & 8,7 & 8,4 & 0,22 & $<0,001$ \\
\hline Freizeit & 7,8 & 7,2 & 0,28 & $<0,001$ \\
\hline Sicherheit & 7,5 & 7,5 & 0,01 & 0,701 \\
\hline Qualität der gesellschaftlichen Organisation & 6,9 & 6,8 & 0,08 & 0,004 \\
\hline Wohnen und natürliche Wohnumgebung & 8,4 & 8,4 & $-0,01$ & 0,840 \\
\hline Subjektives Wohlbefinden & 8,1 & 7,7 & 0,31 & $<0,001$ \\
\hline
\end{tabular}

Quelle: Eigene Berechnungen auf Basis von EU-SILC 2013, Statistik Austria; Globale Signifikanzschwelle $\alpha=0.05$; nach Bonferroni-HolmKorrektur (wegen multiplen Testens) signifikante $p$-Werte fettgedruckt

Tabelle 3: BLI Jugend nach Geschlecht

\begin{tabular}{lcccc}
\hline & $\begin{array}{c}\text { Männlich } \\
(\boldsymbol{n}=\mathbf{1 0 3 1})\end{array}$ & $\begin{array}{c}\text { Weiblich } \\
(\boldsymbol{n = 9 4 5 )}\end{array}$ & $\begin{array}{c}\text { Effect size (Co- } \\
\text { hen's } \boldsymbol{d})\end{array}$ & $\begin{array}{c}\boldsymbol{p} \text {-Wert (t-Test für un- } \\
\text { abh. Stichproben) }\end{array}$ \\
\hline BLI & 78,3 & 78,0 & 0,01 & 0,887 \\
Materielle Lebensbedingungen & 6,8 & 6,7 & 0,06 & 0,158 \\
Produktive Aktivitäten und Arbeit & 8,3 & 8,4 & $-0,05$ & 0,258 \\
Gesundheit & 8,4 & 8,3 & 0,10 & 0,022 \\
Bildung & 5,7 & 6,4 & $-0,23$ & $<0,001$ \\
Soziale Beziehungen & 8,6 & 8,9 & $-0,20$ & $<0,001$ \\
Freizeit & 7,9 & 7,7 & 0,12 & 0,010 \\
Sicherheit & 7,9 & 7,1 & 0,47 & $<0,001$ \\
Qualität der gesellschaftlichen Organisation & 6,8 & 7,0 & $-0,13$ & 0,003 \\
Wohnen und natürliche Wohnumgebung & 8,4 & 8,4 & 0,05 & 0,314 \\
Subjektives Wohlbefinden & 8,1 & 8,1 & $-0,06$ & 0,186 \\
\hline
\end{tabular}

Quelle: Eigene Berechnungen auf Basis von EU-SILC 2013, Statistik Austria; Globale Signifikanzschwelle $\alpha=0.05$; nach Bonferroni-HolmKorrektur (wegen multiplen Testens) signifikante $p$-Werte fettgedruckt (nach der Korrektur erscheinen Freizeit und Gesundheit nicht mehr als signifikante Prädiktoren)

Erwachsenenalters einen Indexwert mit denselben Gewichten berechnet. Zwar ist davon auszugehen, dass Personen des mittleren Erwachsenenalters anderen Aspekten eine hohe Bedeutung für die Lebensqualität zuordnen; um jedoch eine Vergleichbarkeit der Ergebnisse zu gewährleisten, ist die Berechnung eines äquivalenten Index alternativlos.

Sowohl was den Gesamt-BLI als auch was die Subindikatoren außer Arbeit, Sicherheit, Wohnen und Einkommen betrifft (und Bildung, welche trivialerweise für Erwachsene höher ausfällt und deshalb nicht angeführt ist), weisen die Jugendlichen und jungen Erwachsenen signifikant höhere Werte auf als Personen zwischen 30 und 59 (t-Test für unabhängige Stichproben, berechnet anhand gewichteter Daten ). ${ }^{2}$

Entweder ist die Jugendphase tatsächlich für den Großteil der Befragten eine vergleichsweise erfreuliche Zeit, oder Jugendliche neigen generell zu einem positiveren Antwortstil oder vermehrtem Optimismus bei der Bewertung. Insbesondere könnten Einschränkungen wie etwa eine schwierigere materielle Lage im Jugendalter eher als vorübergehend angesehen werden und dadurch weniger gewichtig als im Erwachsenenalter.

2 Berechnungen unter Verwendung der Prozedur Complex Samples in SPSS 


\begin{tabular}{|c|c|c|c|c|c|}
\hline & $\begin{array}{l}\text { dicht besiedelte } \\
\text { Region }(n=582)\end{array}$ & $\begin{array}{c}\text { mittlere Region } \\
(n=607)\end{array}$ & $\begin{array}{c}\text { dünn besiedelte } \\
\text { Region }(n=787)\end{array}$ & $\begin{array}{c}\text { Effect size } \\
\left(\eta^{2}\right)\end{array}$ & $\begin{array}{l}p \text {-Wert, } \\
\text { ANOVA }\end{array}$ \\
\hline BLI & 74,5 & 79,5 & 79,7 & 0.04 & $<0,001$ \\
\hline Materielle Lebensbedingungen & 6,1 & 7,1 & 7,3 & 0.05 & $<0,001$ \\
\hline $\begin{array}{l}\text { Produktive Aktivitäten und } \\
\text { Arbeit }\end{array}$ & 8,0 & 8,6 & 8,6 & 0.02 & $<0,001$ \\
\hline Gesundheit & 8,2 & 8,5 & 8,6 & 0.01 & 0,005 \\
\hline Bildung & 7,4 & 7,0 & 6,1 & 0.05 & $<0,001$ \\
\hline Soziale Beziehungen & 8,5 & 8,9 & 8,9 & 0.01 & $<0,001$ \\
\hline Freizeit & 7,3 & 8,1 & 8,1 & 0.02 & $<0,001$ \\
\hline Sicherheit & 6,8 & 7,5 & 8,0 & 0.07 & $<0,001$ \\
\hline $\begin{array}{l}\text { Qualität der gesellschaftlichen } \\
\text { Organisation }\end{array}$ & 6,7 & 6,9 & 7,1 & 0.01 & $<0,001$ \\
\hline $\begin{array}{l}\text { Wohnen und natürliche Wohn- } \\
\text { umgebung }\end{array}$ & 7,8 & 8,5 & 8,7 & 0.06 & $<0,001$ \\
\hline Subjektives Wohlbefinden & 7,8 & 8,3 & 8,3 & 0.02 & $<0,001$ \\
\hline
\end{tabular}

Quelle: Eigene Berechnungen auf Basis von EU-SILC 2013, Statistik Austria; Globale Signifikanzschwelle $\alpha=0.05$; nach Bonferroni-HolmKorrektur (wegen multiplen Testens) signifikante $p$-Werte fettgedruckt

\subsection{Geschlecht}

Nach Geschlecht zeigt sich hingegen ein durchaus differenziertes Bild: Zwar gibt es, was den Gesamtindex betrifft, keine signifikanten Unterschiede zwischen jungen Frauen und jungen Männern, allerdings weisen, wie in Tabelle 3 zu sehen ist, Frauen im Bereich Sicherheit signifikant niedrigere Werte auf. Beim Subindex Sicherheit lassen sich diese auf niedrigere Werte beim Item „Wie sicher fühlen Sie sich, wenn Sie nach Einbruch der Dunkelheit alleine zu Fuß in Ihrer Wohngegend unterwegs sind?" zurückführen. Junge Männer hingegen erreichen bezüglich der sozialen Beziehungen, der wahrgenommenen Qualität der gesellschaftlichen Organisation und der Bildung niedrigere Werte.

\subsection{Stadt und Land}

Wie in Abschnitt 2.3 bereits erwähnt, hatten die Autor/ inn/en des Jugendberichts zunächst angenommen, dass es sich bei Jugendlichen in ländlichen Regionen um „regional benachteiligte" Jugendliche handelt. Doch nicht nur, dass die Jugendlichen der entsprechenden Fokusgruppe meinten, dass es schön sei, dass sich einmal jemand für sie interessiere, dass sie sich aber keinesfalls benachteiligt fühlten und die Schönheit ihres Lebensraumes sehr schätzten; auch in der quantitativen Auswertung zeigt sich - wie in Tabelle
$4 \mathrm{zu}$ sehen ist -, dass Jugendliche in ländlicheren Regionen im Gesamtindex und fast allen Subindizes signifikant höhere Werte aufweisen als Jugendliche und junge Erwachsene in dichter besiedelten Regionen (einfaktorielle ANOVA auf Basis gewichteter Daten). Nur bei der Bildung zeigt sich ein umgekehrter Effekt, was unter anderem mit der schlechteren Erreichbarkeit von weiterführenden Bildungsinstitutionen (aber auch Selbstselektionseffekten - Studierende ziehen oft in die Städte) zusammenhängen dürfte.

Nun ist allerdings bekannt, dass sich die Bevölkerungsstrukturen von Stadt und Land deutlich unterscheiden: Nicht nur ist die Armut in den Städten höher (siehe z.B. Statistik Austria 2014/2015: 68), auch leben dort mehr Menschen mit ausländischer Staatsbürgerschaft (siehe z.B. Statistik Austria 2017: 81). Es zeigt sich allerdings, dass Unterschiede auch dann noch bestehen bleiben, wenn derartige demographische Einflüsse herausgerechnet werden (BMFJ 2016: 62). Dies legt nahe, dass tatsächlich spezifische Lebensbedingungen am Land wie eben der leichtere Zugang zu Grünraum und Natur und ein stärkerer sozialer Zusammenhalt relevante Faktoren für verschiedene Aspekte der Lebensqualität darstellen.

\section{Rezeption und Kritik}

Nach Abschluss der Arbeiten am Jugendbericht stellten die Autor/inn/en die Ergebnisse auf verschiedenen 
nationalen und internationalen Konferenzen vor und waren darüber hinaus als Expert/inn/en bei der parlamentarischen Behandlung des Berichts im Familienausschuss geladen. Die in den jeweiligen Diskussionen behandelten Punkte werden in den nächsten Abschnitten diskutiert.

\subsection{Quantitative Messbarkeit von Lebensquali- tät}

Bei der parlamentarischen Behandlung des Jugendberichts im Familienausschuss äußerte der ebenfalls als Experte geladene Sozialwissenschaftler Philipp Ikrath Zweifel daran, dass sich subjektive Befindlichkeiten rein quantitativ erheben lassen (Parlament der Republik Österreich 2017). Tatsächlich sind quantitative Maße der Lebensqualität nicht ohne Kritik geblieben, welche eine langjährige Diskussion über die Qualität von subjektiven Daten ausgelöst hat (für einen Überblick siehe etwa Angner 2005, Haybron 2008, Schwarz et al. 2008 oder Ponocny et al. 2005). Thematisiert werden unter anderem die prinzipielle Messbarkeit, die zeitliche Stabilität und die Bedeutung des aktuellen Befragungssettings, die Bedeutung und Dimensionalität von subjektivem Wohlbefinden sowie die Skalierung.

Trotzdem kann gesagt werden, dass es sich bei der (quantitativ erfassten) allgemeinen Lebenszufriedenheit um ein gut etabliertes und weit verbreitetes Maß zur Erfassung von Lebensqualität handelt, welches es ermöglicht, reliable und valide Aussagen über Populationen zu treffen (Diener et al. 2013). Inzwischen ist die Erfassung der allgemeinen Lebensqualität auch in der amtlichen Statistik verbreitet, die lange ein eher distanziertes Verhältnis zu subjektiven Maßen dieser Art hatte, insbesondere in wirtschaftspolitischen Zusammenhängen (siehe z.B. Bache/Reardon 2016:, 153). Der Mehrwert durch allfällige qualitative Zusatzerhebungen soll dadurch aber nicht in Abrede gestellt werden.

\subsection{Prinzipielle Sinnhaftigkeit von zusammen- gesetzten Indikatoren}

Ein Kritikpunkt, der auf einer der Konferenzen aufgeworfen wurde, war der der Problematik zusammengesetzter Indikatoren. In einem Index wie dem zu konstruierenden BLI Jugend werden Variablen verschiedener Art aus verschiedenen Lebensbereichen miteinander $\mathrm{zu}$ einem composite indicator verrechnet. Ein derartiges Vorgehen ist gut etabliert und führt $\mathrm{zu}$ leicht kommunizierbaren Resultaten (siehe etwa Mag-
gino/Zumbo 2012). Dadurch können allerdings Unterschiede in einzelnen Dimensionen verdeckt werden: Sollte eine Gruppe in einem Lebensbereich oder hinsichtlich einer bestimmten Variable besonders niedrige Werte aufweisen, in einem anderen Lebensbereich jedoch besonders hohe, so wäre ein Durchschnittsindex über diese beiden Bereiche nicht in der Lage, das Problemfeld $\mathrm{zu}$ identifizieren. Tatsächlich ist dieses Kompensieren ein nicht zu unterschätzendes Problem aggregierter Indikatoren, auf das beispielsweise auch Freudenberg (2003) und Maggino/Zumbo (2012) hinweisen. Freudenberg (2003: 29) empfiehlt dementsprechend, sie mit zusätzlichen Informationen und einer genauen Beschreibung ihrer Konstruktion zu ergänzen sowie zu überprüfen, wie robust sie sich gegenüber methodischen Veränderungen verhalten. Eine solche Überprüfung der Robustheit wurde tatsächlich durchgeführt, wie im Abschnitt 2.5. beschrieben. Weiters muss auch darauf hingewiesen werden, dass bei der Verwendung des BLI Jugend im Rahmen des 7. Berichts zur Lage der Jugend gerade die Subindizes betrachtet wurden.

Darüber hinaus wurde die Kritik geäußert, dass durch einen Index eigentlich (wie bei einer Skala in der Psychologie) eine zugrundeliegende latente Variable gemessen würde oder zumindest gemessen werden sollte (in diesem Fall so etwas wie die allgemeine Lebenszufriedenheit), was wiederum einen faktorenanalytischen Ansatz nahelegen würde. Hier muss jedoch auf die Unterscheidung zwischen reflexiven Indikatoren, die in der Tat latente Variablen abzubilden versuchen, und formativen Indikatoren hingewiesen werden. Bei Letzteren geht es nicht um die Messung einer latenten Variablen, weshalb die einzelnen eingehenden Variablen auch nicht notwendigerweise miteinander korrelieren. Diese werden vielmehr als definierende Bestandteile einer interessierenden Variablen wie etwa der Lebensqualität angesehen (siehe z.B. Mauro et al. 2018, 76). Entsprechend ist der BLI Jugend als formativer und eben nicht als reflexiver Indikator aufzufassen.

\subsection{Berücksichtigung besonderer Gruppen}

Eine Kritik am Bericht aus den Reihen der Parlamentarier/innen war, dass bestimmte Gruppen wie zum Beispiel junge Menschen mit Behinderung oder junge Menschen in Haft im Index und im Bericht generell nicht repräsentiert seien. Junge Menschen mit Behinderung kommen aber durchaus im Bericht vor: Sie 
waren sowohl in den Expert/inn/engruppen als auch in den spezifischen Analysen des BLI (gemessen über dauerhafte gesundheitliche Beeinträchtigung) vertreten. Grundsätzlich ist das aber ein valider Kritikpunkt: Quantitative Analysen, die ein repräsentatives Abbild einer Population zum Ziel haben, ermöglichen grundsätzlich vor allem Aussagen über den durchschnittlichen Repräsentanten/die durchschnittliche Repräsentantin dieser Gruppe sowie über große Subgruppen. Kleine Subgruppen, wie eben zum Beispiel Jugendliche in Haft, sind in einer Stichprobenerhebung nicht oder mit so wenigen Personen vertreten, dass keine Aussagen speziell über sie getroffen werden können. ${ }^{3}$ Stehen solche Spezialgruppen im Fokus bestimmter jugendpolitischer Maßnahmen, dann sollten andere Herangehensweisen gewählt werden, um die Zielgruppe generell $\mathrm{zu}$ erforschen und die Wirksamkeit bestimmter Maßnahmen zu evaluieren. Eine andere Möglichkeit wäre, Gruppen, an denen ein spezielles Interesse besteht, zu oversamplen.

\section{Diskussion und Conclusio}

Beim Better-Life-Index Jugend handelt es sich um ein methodisch durchaus solide konstruiertes Instrument, das sich allerdings, wie in Abschnitt 5.4 beschrieben, nur für einen Teil der möglichen Anwendungsfelder tatsächlich eignet. Mit seiner Hilfe lassen sich Risikogruppen mit besonders niedriger Lebensqualität insgesamt oder in besonderen Bereichen identifizieren bzw. auch Bereiche, in welchen diese Gruppen besonders schlechte Werte aufweisen. Außerdem setzt er durch seine Konstruktion einen Schwerpunkt auf jene Aspekte, welche speziell für Jugendliche und junge Erwachsene von Belang sind. Ein internationaler Vergleich, ein Vergleich des Niveaus der Lebensqualität in den verschiedenen Bereichen und eine Veränderungsmessung sind zur Zeit allerdings nicht möglich, wie im Folgenden ausgeführt wird.

\subsection{Evaluierung politischer Maßnahmen}

Um die Wirksamkeit politischer Maßnahmen zu evaluieren, muss ein entsprechendes Messinstrument mehrfach anwendbar sein (idealerweise vor und nach der

3 Bei EU-SILC gibt es noch dazu die Besonderheit, dass es sich um eine Haushaltserhebung handelt und keine Personen in Anstaltshaushalten, also auch Jugendheimen und Gefängnissen, vertreten sind.
Maßnahme), um die erwarteten Veränderungen überhaupt erfassen zu können. Der BLI Jugend in seiner für den 7. Jugendbericht entwickelten Form beruht jedoch zu einem nicht unwesentlichen Teil auf Daten, die nur für 2013 verfügbar sind (Daten des EU-SILCSondermoduls Well-being), aber aller Voraussicht nach nur teilweise in den Folgejahren. So werden nur 18 der insgesamt 41 im Index verwendeten Items und Variablen jährlich erhoben, und Bereiche wie die Qualität der gesellschaftlichen Organisation oder soziale Beziehungen könnten mit den jährlichen Standarddaten überhaupt nicht abgebildet werden. Grundsätzlich wäre es möglich, die fehlenden Variablen regelmäßig über eine Zusatzstudie zu erheben und diese dann mittels Statistical Matchings mit EU-SILC zu verknüpfen, wobei darauf $\mathrm{zu}$ achten wäre, geeignete Linkvariablen vorzusehen, die auch die interessierenden Sub- und Risikogruppen abbilden. Hierbei könnten Zusatzerhebung und Index so angepasst werden, dass auch bislang nicht berücksichtigte Bereiche und Themenfelder, die in den Fokusgruppen als wichtig angemahnt wurden, miterfasst werden.

Aber auch wenn eine Wiederholbarkeit der Berechnung und damit die Möglichkeit eines regelmäßigen Monitorings gegeben wäre, müsste ein entsprechender Index spezifisch und sensibel genug sein, um die erwarteten Veränderungen überhaupt erfassen zu können. Die Spezifität und Sensibilität kann unabhängig von der konkreten Maßnahme nicht bewertet werden, allgemein lässt sich aber festhalten, dass der BLI Jugend nur dort spezifisch sein kann, wo EU-SILC besonders detaillierte Informationen erhebt, was zum Beispiel beim Einkommen und Wohnen, 2013 aber auch bei der subjektiven Lebensqualität der Fall ist. Darüber hinaus ist zu fragen, ob die Zielgruppe der Maßnahme groß genug ist, um in der Stichprobe ausreichend repräsentiert zu sein, was bei konkreten risikogruppenspezifischen oder auch regionalen Maßnahmen in der Regel nicht der Fall sein dürfte. So können anhand von EUSILC wahrscheinlich durchaus Aussagen über die Lage junger Frauen getroffen werden, nicht aber über die Lebensqualität von migrantischen Wiener/inne/n der zweiten Generation oder von jungen Haftentlassenen.

Schließlich ist bezüglich der Sensitivität (vor allem der Messbarkeit von Veränderungen) zu berücksichtigen, dass vor allem Maße der subjektiven Zufriedenheit oft wenig änderungssensitiv sind. Adaptationsprozesse führen häufig dazu, dass sich die Zufriedenheitswerte nach einiger Zeit wieder den Ausgangswerten vor der Veränderung angleichen (für eine Diskussion siehe 
etwa Diener 2000). Eine gleichzeitige Verbesserung der Lebensbedingungen eines großen Teils der Zielbevölkerung zu einem Zeitpunkt sollte demnach also zunächst zu einer Erhöhung des BLI führen, mit langsamem Absinken bei zunehmendem zeitlichen Abstand zur Maßnahme oder zum gesellschaftlichen Ereignis. Eine Ausnahme bildet hier Arbeitslosigkeit, für die sich keine Adaptation nachweisen lässt und die auch nach ihrer Beendigung weiterhin negative Auswirkungen auf die Zufriedenheit zeitigt (siehe Dolan et al. 2008).

Zusammenfassend kann gesagt werden, dass dort, wo eine evidenzbasierte Jugendpolitik Informationen über die Wirksamkeit einer konkreten (zielgruppenspezifischen) Maßnahme benötigt, andere Erhebungsinstrumente und Designs in der Regel geeigneter sein werden als der BLI Jugend.

\subsection{Gesellschaftliche Aufmerksamkeit für Jugendagenden}

Großangelegte sozialwissenschaftliche Studien, die idealerweise internationale Rankings oder Vergleiche erlauben, lenken die (mediale und öffentliche) Aufmerksamkeit auf den entsprechenden Gegenstand bzw. die entsprechende Zielgruppe. Ein gutes Beispiel dafür ist die PISA-Erhebung, die durch jeweils schlechte Rankingpositionen im internationalen Vergleich die Aufmerksamkeit auf alltagsrelevante Fähigkeiten von Jugendlichen und damit auf Leistungen oder Defizite des Schulsystems gelenkt hat und dadurch sehr Policywirksam geworden ist (siehe z.B. Breakspear 2012).

Nun ergeben sich beim BLI Jugend allerdings nur beschränkt Rankingmöglichkeiten. Zwar wurde 2013 in allen SILC-Ländern ein Sondermodul zum Thema Lebensqualität durchgeführt, da in der österreichischen Erhebung jedoch eine ganze Reihe von nationalen Zusatzvariablen erfragt wurde, die auch ihren Niederschlag im BLI Jugend fanden, lässt sich der BLI Jugend in seiner momentanen Form nicht international nachbilden. Variablen wie Autonomie oder die Zufriedenheit mit der verfügbaren Zeit für bestimmte Personen oder Dinge wären nicht in allen Ländern vorhanden und die Gewichtung der übrigen Variablen müsste angepasst werden. Hinzu kommt, dass es fraglich ist, ob die Gewichtung durch österreichisch besetzte Expert/inn/engruppen dann überhaupt vertretbar wäre.

Da derzeit weder ein internationaler noch ein Vergleich zwischen verschiedenen Lebensbereichen (siehe Abschnitt 5.4) möglich ist, bleibt nur der Vergleich unterschiedlicher Subgruppen, was aber in weiten Bereichen zu durchaus vorhersagbaren Ergebnissen geführt hat. Eine Ausnahme stellt hier die überraschend hohe Lebensqualität bei jungen Menschen am Land dar, was aber alleine noch nicht ausreichen dürfte, um dem Index Aufmerksamkeit zu verschaffen.

Tatsächlich hat dann die Veröffentlichung des BLI Jugend mit Ausnahme einer Pressemitteilung des parlamentarischen Pressediensts unserer Kenntnis nach kaum mediales Echo erfahren, was aber im Kontext der Pressepolitik der BMFJ und der beteiligten Institute und Einrichtungen bewertet werden muss. Generell muss natürlich zwischen medialer Aufmerksamkeit und der tatsächlichen Implementierung von PolicyMaßnahmen unterschieden werden.

5.3 Erkenntnisse darüber, was für Jugendliche ein gutes Leben ausmacht

Während sich die vorherigen Punkte auf den Index und seine Analysemöglichkeiten bezogen haben, geht es hier um den Konstruktionsprozess und die Erkenntnisse, die sich daraus ziehen lassen. Wie in Abschnitt 2.5 gezeigt, führen unterschiedliche Gewichtungen bei den Variablen zu kaum unterschiedlichen Ergebnissen, vor allem nicht was Gruppenunterschiede betrifft, die ja im Fokus der BLI-Analysen stehen. Der Entscheidungsprozess selbst hat aber die unterschiedliche Bedeutsamkeit verschiedener Lebensbereiche und Einzelfaktoren für die Lebensqualität von jungen Menschen sichtbar gemacht, etwa was Ruhe und ein hohes persönliches Einkommen im Vergleich zu Gesundheit, Glück und Autonomie angeht. Die Dokumentation der entsprechenden Datenlücken und damit der Mängel des BLI geben nicht nur Aufschluss, welche Lebensbereiche in einem Index mit eigener Erhebung noch erfasst werden sollten, sondern liefern darüber hinaus wichtige Informationen darüber, was für Jugendliche und junge Erwachsene ein lebenswertes Leben ausmacht.

\subsection{Identifikation von Problemfeldern}

Die aus Gruppenvergleichen bezüglich des BLI erhaltenen Informationen können dabei helfen, Politikmaßnahmen zielgerichtet auf bestimmte Personen zuzuschneiden. So könnte das Ergebnis, dass junge Frauen sich weniger sicher fühlen und dass dies auf mangelndes Sicherheitsempfinden nachts in der Wohnumgebung zurückgeht, dazu beitragen, spezielle 
Maßnahmen zum Beispiel zur Gestaltung von Wohnanlagen, Gehwegen, Unterführungen und Parks zu entwickeln, um Sicherheit und/oder Sicherheitsempfinden ${ }^{4}$ junger Frauen zu verbessern. Um herauszufinden, welche Maßnahmen genau zum angestrebten Ziel führen könnten, bedarf es dann allerdings jeweils weiterer Forschung. Dies gilt sowohl für die detailliertere Erfassung des Problems wie möglicherweise auch in Bezug auf die Stichprobengröße, wo etwa regionale kleinräumige Schlussfolgerungen aufgrund zu geringer Zellenbesetzungen nicht mehr möglich sind.

Was der Index jedoch prinzipiell nicht leisten kann, ist die Identifikation von Bereichen, in denen Jugendliche und junge Erwachsene in Österreich verglichen mit anderen Lebensbereichen eine besonders niedrige Lebensqualität aufweisen. Dies liegt in der Art der Konstruktion des Index begründet: Da die Subindizes aus Variablen mit ganz unterschiedlichen Antwortskalen und Antwortverteilungen bestehen, können die einzelnen Subindizes in ihrem Niveau nicht miteinander verglichen werden.

\subsection{Ausblick}

Was die Vergleichbarkeit über die Zeit und zwischen verschiedenen Ländern angeht, könnten Zusatzerhebungen Abhilfe schaffen, die über Statistical Matching an den jährlich verfügbaren Basisdatensatz von EUSILC angebunden werden. Diese Erhebung könnte auch Zufriedenheitsitems zu allen zehn Lebensbereichen enthalten, was den (vom BLI Jugend getrennten) Vergleich der Zufriedenheiten mit den verschiedenen Lebensbereichen ermöglichen würde. Eventuell könnte dabei teilweise auch auf bestehende Erhebungen zurückgegriffen werden. So wurde zum Beispiel in der letzten Gesundheitsbefragung 2014 das Lebensqualitätsinstrument der WHO, der WHOQOL (WHO 1998) in der Kurzform (WHOQOL BREF) mitgeführt, der unter anderem Items zur Zufriedenheit mit Beförderungsmitteln, mit dem Sexualleben und mit dem eigenen Aussehen enthält. Eine Verknüpfung von EU-SILC

4 Maßnahmen, die lediglich darauf abzielen, das Sicherheitsempfinden zu verbessern, ohne die tatsächliche Sicherheit zu erhöhen, mögen zynisch erscheinen. Es ist aber zu berücksichtigen, dass ein erhöhtes rein subjektives Sicherheitsempfinden nicht nur $\mathrm{zu}$ einer höheren allgemeinen Lebensqualität beitragen, sondern auch dazu führen kann, dass die betreffenden Personen die entsprechenden Räume und Einrichtungen häufiger nutzen, was dann wiederum ihre Sicherheit erhöhen kann. mit diesen Daten aus der Gesundheitsbefragung könnte hier zu einem vollständigeren Bild der Lebensqualität der jungen Bevölkerung führen. Dabei ist allerdings zu beachten, dass auch die Gesundheitsbefragung nur in größeren Abständen durchgeführt wird und nicht sicher ist, ob sie den WHOQOL BREF beim nächsten Mal wieder enthält.

Da sich, wie in Abschnitt 2.5 beschrieben, bezüglich der allgemeinen Lebenszufriedenheit aus EU-SILC ganz ähnliche Gruppenunterschiede und damit Risikogruppen zeigen wie bezüglich des BLI Jugend (und mit anderen Gewichten konstruierte vergleichbare Indizes), wäre auch zu diskutieren, ob dieses einfache und damit einfach zu kommunizierende und noch dazu regelmäßig erhobene Maß nicht ausreicht, um die Lebensqualität von jungen Menschen in Österreich regelmäßig zu erfassen. Hierdurch könnten laufend sowohl verschiedene Gruppen junger Menschen als auch verschiedene Zeitpunkte miteinander verglichen werden. Benötigt man zusätzlich Informationen über verschiedene Lebensbereiche, könnte man darüber hinaus regelmäßig die Zufriedenheit mit verschiedenen Lebensbereichen erheben (für Wohnen, Einkommen und Arbeit bereits in der österreichischen SILC-Erhebung enthalten). Hierbei ist allerdings, wie in 5.1 erwähnt, zu berücksichtigen, dass Adaptationsprozesse bei der Lebenszufriedenheit objektive Verbesserungen oder Verschlechterungen der Lebensbedingungen verschleiern könnten. Außerdem erlauben solche globalen Maße keine Rückschlüsse auf Faktoren, die zu dieser Einschätzung beitragen und damit auf konkrete politische Handlungsfelder. Bezüglich des internationalen Vergleichs - unter der Annahme, hier könnte eine entsprechende Datenbasis geschaffen werden - muss zusätzlich berücksichtigt werden, dass subjektive Items aufgrund der Übersetzung (bzw. nicht exakten Übersetzbarkeit) in andere Sprachen, aber auch aufgrund kultureller Unterschiede nicht in jedem Land dasselbe messen und die entsprechenden Ergebnisse damit nur bedingt international vergleichbar wären. Und schließlich ist nicht sicher, ob das, was für Gruppenunterschiede innerhalb eines österreichischen Datensatzes gilt, nämlich, dass sie von einem einfachen Maß wie der Lebenszufriedenheit genauso gut abgebildet werden können wie von einem komplexen Index, auch für Länderunterschiede gilt. Hier könnte ein Index, der stärker auf objektiven Variablen (wie etwa Einkommen, Arbeitslosigkeit oder Ausbildungsstatus) basiert, zu einem doch deutlich anderen Länderranking führen als eine subjektive Maßzahl. 
Ein weiterer Ausbau des BLI Jugend würde aber angesichts des zu finanzierenden Aufwands - auch eine klare politische Vorgabe erfordern, mit genauen Vorstellungen darüber, welchen Zwecken ein solcher Index im politischen Prozess dienen soll. Wie gezeigt, können der Better-Life-Index Jugend in seiner derzeitigen Form bzw. die bei seiner Konstruktion gewonnenen Erkenntnisse immerhin einen Ausgangspunkt für die Diskussion über Einsatzmöglichkeiten und Einsatzzwecke entsprechender Indizes bilden, die Eignung als Policy-Instrument unterliegt zumindest derzeit jedoch wesentlichen Einschränkungen.

\section{Literatur}

Angner, E. (2005): Is it possible to measure happiness? The measurement-theoretic argument against subjective measures of wellbeing. Online: http://citeseerx.ist.psu.edu/ viewdoc/download?doi=10.1.1.92.7559\&rep=rep1\&type $=$ pdf [21.04.2015].

Babic, B. (2017): Well-being. (K)ein Thema für die Jugendforschung in Österreich. Vortrag, gehalten bei der Konferenz „Jugend-Lebenswelt-Bildung - Perspektiven der Jugendforschung in Österreich" 16.-18.11.2017, Innsbruck.

Bache, I./Reardon, L. (2016): The politics and policy of wellbeing. Understanding the rise and significance of a new agenda. Cheltenham: Edward Elgar Publishing.

BMFJ (2016): 7. Bericht zur Lage der Jugend in Österreich. Teil B: Better-Life-Index Jugend. Wien. Online: https://www. frauen-familien-jugend.bka.gv.at/dam/jcr:a49a222caf66-40oe-ao2f-7f65d672afid/7.\%20Jugendbericht $\% 20$ Teil\%2oB\%2ofinal.pdf [11.12.2018].

Bradshaw, J./ Hoelscher, P/ Richardson, D. (2007): Comparing Child Well-Being in OECD Countries: Concepts and Methods. Innocenti Working Paper No. 2006-03. Florence, UNICEF Innocenti Research Centre.

Breakspear, S. (2012): „The Policy Impact of PISA: An Exploration of the Normative Effects of International Benchmarking in School System Performance“. OECD Education Working Papers, No. 71, OECD Publishing. Online: http://dx.doi.org/10.1787/5kgfdfqffr28-en [28.04.2018].

Council of Europe (2008): 8th Council of Europe Conference of Ministers responsible for Youth, „The future of the Council of Europe youth policy: AGENDA 2020" declaration, online: Online: https://rm.coe.int/1680702429 [12.11.2018].

Dell'Aglio, D./Cunningham, W./Koller, S./Borges, V. C./Leon, J. S. (2007): Youth well-being in Brazil: an index for crossregional comparisons. World Bank Policy Research Working Paper (4189).

Denstat, F. Y. (2009): Youth Policy Manual. How to develop a national youth strategy. Strasbourg: Council of Eu- rope Publishing. Online: https://pjp-eu.coe.int/documents/1017981/7110707/YP_Manual_pub.pdf/7b17e1e6e8b6-4041-9o2e-3b3ado973c45 [12.11.2018].

Diener, E. (2000): Subjective well-being: The science of happiness and a proposal for a national index. American psychologist, 55 (1), 34-43.

Diener, E./Inglehart, R./Tay, L. (2013): Theory and validity of life satisfaction scales. Social Indicators Research, 112 (3), 497-527.

Dolan, P./Peasgood, T./White, M. (2008): Do we really know what makes us happy? A review of the economic literature on the factors associated with subjective well-being. Journal of economic psychology, 29 (1), 94-122.

European Commission (2009): Mitteilung der Kommission, Eine EU-Strategie für die Jugend - Investitionen und Empowerment, Eine neue offene Methode der Koordinierung, um auf die Herausforderungen und Chancen einzugehen, mit denen die Jugend konfrontiert ist. $\mathrm{KOM}(2009) 200$ endgültig, Brüssel.

Eurostat (2011): Sponsorship Group on Measuring Progress, Well-being and Sustainable Development. - Final Report adopted by the European Statistical System Committee. Online: http://ec.europa.eu/eurostat/web/ess/about-us/ measuring-progress [28.04.2018].

Foundation for Child Development (2013): National Child and Youth Development Index (CWI). Online: http://fcdus.org/sites/default/files/FINAL\%2O2012\%20CWI\%20 Report.pdf [11.08.2018].

Freudenberg, M. (2003): Composite indicators of country performance. A critical assessment. STI working paper, 2003/16, Industry Issues. Paris: OECD. Online: http:// www.oecd.org/officialdocuments/publicdisplaydocum entpdf $/$ ? cote $=$ DSTI $/$ DOC $(2003) 16 \&$ docLanguage $=$ En [28.04.2018].

Goldin, N. (2014): The global youth wellbeing index. Lanham, Rowman \& Littlefield.

Haybron, D. M. (2008): Philosophy and the science of subjective well-being. In: Eid, M./ Larsen, R. J. (Hg): The science of subjective well-being: Critical perspectives. New York: Guilford Press, 17-43.

Keul, A.G./Brunner, B./Blaschke, T. (2017): Städtische Lebensqualität: Zur Systematik objektiver und subjektiver Deskriptoren. Momentum Quarterly, 6 (2), 63-152.

Land, K. C./Lamb, V. L./Mustillo, S. K. (2001): Child and youth well-being in the United States, 1975-1998: Some findings from a new index. Social indicators research, 56 (3), 241-318.

Maggino, F./Zumbo, B.D. (2012): Measuring the quality of life and the construction of social indicators. In: Land, K.C./ Michalos, A.C./Sirgy, J. (Hg.): Handbook of social indicators and quality of life research. Dordrecht: Springer, 201-238.

Mauro, V./Biggeri, M./Maggino, F. (2018): Measuring and monitoring poverty and well-being: A new approach for the synthesis of multidimensionality. Social Indicators Research, 135, 75-89. Online: https://doi.org/10.1007/ s11205-016-1484-1 [28.04.2018]. 
Noll, H. H. (2004): Social indicators and Quality of Life research: Background, achievements and current trends. In: N. Genov (2004): Advances in sociological knowledge. Wiesbaden: VS Verlag für Sozialwissenschaften, 151-181.

OECD (2011): How's life? Measuring Well-Being. OECD Publishing. Online: http://dx.doi.org/10.1787/9789264121164en [28.04.2018].

OECD (2015): Better Life Index. Online: http://www.oecdbetterlifeindex.org/de/ [28.04.2018].

Parlament der Republik Österreich (2017): Familienausschuss: Expertenhearing zum Bericht der Lage der Jugend. Parlamentskorrespondenz Nr. 56 vom 25.1.2017. Online: https://www.parlament.gv.at/PAKT/PR/JAHR_2017/ PKo056/ [02.04.2018].

Ponocny, I./Weismayer, C./Stross, B./Dressler, S. G. (2016): Are most people happy? Exploring the meaning of subjective well-being ratings. Journal of Happiness Studies, 17 (6), 2635-2653.

Schneider, U./Kleindienst, J. (2016): Monetising the provision of informal long-term care by elderly people: estimates for European out-of-home caregivers based on the wellbeing valuation method. Health and Social Care in the Community, 24 (5), 81-91.

Schwarz, N./ Knäuper, B./ Oyserman, D./ Stich, C. (2008): The psychology of asking questions. In: Leeuw, E. de/ Hox, J./Dillman, D. (Hg.): International handbook of survey methodology. New York: Taylor \& Francis, 18-34.

Statistik Austria (2014): Standarddokumentation Metainformationen zu EU-SILC. Wien. Online: http://www.statistik.at/wcm/idc/idcplg?IdcService=GET_PDF_FILE \&RevisionSelectionMethod=LatestReleased\&dDocNa $\mathrm{me}=102699$ [28.04.2018].

Statistik Austria (2014/2015): Tabellenband EU-SILC 2013. Einkommen, Armut und Lebensbedingungen. Wien am 22.10.2014 (Rev. 4 vom 13.8.2015).

Statistik Austria (2016): Wie geht's Österreich? Indikatoren und Analysen. Wien.

Statistik Austria (2017): Migration und Integration 2017. Zahlen. Daten. Fakten. Wien.

Stiglitz, J. E./Sen, A./Fitoussi, J. P. (2010): Report by the commission on the measurement of economic performance and social progress. Paris, Commission on the Measurement of Economic Performance and Social Progress.

UNICEF Office of Research (2013): Child Well-being in Rich Countries: A comparative overview, Innocenti Report Card 11, UNICEF Office of Research, Florence.

WHO (1998): WHOQOL user manual: Programme on mental health. World Health Organization. 